Journal of Computer Science 7 (9): 1353-1357, 2011

ISSN 1549-3636

(C) 2011 Science Publications

\title{
Efficient Feature Extraction Methodology using Wavelet Transform for the Development of Person Identification System using Iris Biometrics
}

\author{
${ }^{1}$ Sulochana Sonkamble and ${ }^{2}$ Ravindra Thool \\ ${ }^{1}$ Department of Information Technology, \\ Marathwada Mitra Mandal's, College of Engineering, Pune, India \\ ${ }^{2}$ Department of Information Technology, \\ Shri Guru Gobind Singji, Institute of Technology, Nanded, India
}

\begin{abstract}
Problem statement: We proposed a new method for feature extraction of an Iris image for personal identification system. Approach: This study purposed a new approach to extract the features of region of interest using the wavelet transform. The extracted Iris region could be normalizing into a rectangular block of fixed dimensions. The Gabor wavelet transform techniques had been applied on the data set to get feature vectors of an iris used for recognition. Results: Two Iris templates used for testing. The system performance score results in a false accept and false reject rate. Conclusion: The performance of the system "Efficient Feature Extraction Methodology Using wavelet transform for the development of personal identification system using iris biometrics" can be increased by training the more feature vectors of an extracted Iris images.
\end{abstract}

Key words: Iris normalization, feature extraction, Gabor wavelet transform, person identification, iris biometrics, transform techniques, feature vectors, iris region, recognition system, proposed system

\section{INTRODUCTION}

Today's world is becoming more dependent on computer based systems. In the computerized world the iris biometrics is the new technology which is playing an important role for the computer based security systems. The iris recognition is becoming a fundamental component of the computerized world with various application areas in national ID card, banking, passport, credit cards, smart cards, PIN, access control and network security (Sonkamble et al., 2008). The biometrics is the study of physical characteristics or behavioral characteristics of human include items such as finger prints, face, hand geometry, voice and iris. Among the biometrics, iris has highly accurate and reliable characteristics. An iris has unique structure and it remains stable over a person life time, which is observed through the clinical evidence (Lewis, 2002).

The ID cards, secret password and PIN are used for personal identification. These can be stolen; passwords can be forgotten or cracked (Ma et al., 2002). The iris biometrics recognition overcomes all the above. Additional convenient security barriers are needed as our society gets more computer dependent and it is needed to have a high confidence secure technology to cope with drawbacks and pitfalls of above technology (Khan et al.,
2004; Masek, 2003). The biometrics is only the authentication for protection of information data ensuring only authorized users are able to access the information on internet (Sonkamble et al., 2010). They are required the person being authenticated to be present at the point of authentication. Thus iris biometrics systems are high confidential secure systems.

The performance of iris recognition system depends on the good image quality and extremely clear iris texture details. We are thankful to CASIA that they have provided the availability of carefully designed iris image database of sufficient size for our experiments ( $\mathrm{Li}$ and Kang, 2008). This study is divided into five sections titled, Introduction, Materials and Methods, Results and Discussions, Conclusion and References.

\section{MATERIALS AND METHODS}

The first and important stage of iris recognition is to isolate the actual iris region in a digital eye. The iris image contains the parts such as pupil, iris, sclera, eyelid (Wang and Han, 2005). So the captured iris image cannot be used directly. The preprocessing is performed after the image captured to isolate the region of interest. The iris region can be divided into two circles, outer for iris-

Corresponding Author: Sulochana Sonkamble, Department of Information Technology, Marathwada Mitra Mandal's, College of Engineering Pune, India 
sclera boundary, another inner for iris-pupil boundary (Proenca and Alexandre, 2006; Delac and Grgic, 2004).

A gradient-based algorithm is developed to overcome the limitations to increase the accuracy of the iris image segmentation compared with existing methods. The magnitude of the gradient values is calculated using these horizontal and vertical values and finding the square root of the combined values. Smooth the iris image with a Gaussian Low pass Filter to reduce noise and unwanted data using the Eq. 1. The Eq. 2 is used to calculate the value of $\mathrm{G}^{\sigma}$ :

$\mathrm{G}(\mathrm{x}, \mathrm{y})=\mathrm{G} \sigma(\mathrm{x}, \mathrm{y}) \mathrm{f}(\mathrm{x}, \mathrm{y})$

Where:

$\mathrm{G} \sigma=\left(1 \frac{1}{\sqrt{2 \Pi \sigma^{2}}}\right) \mathrm{e}^{\left[\left(\mathrm{x}^{2}+\mathrm{y}^{2}\right) / 2 \sigma\right]}$

Compute gradient of $\mathrm{g}(\mathrm{X}, \mathrm{Y})$ using the gradient operator Sobel. The Sobel operator uses a pair of $3 \times 3$ convolution masks, one to calculate the gradient in the $\mathrm{x}$-direction and the other to calculate the gradient in the y-direction. The gradient magnitude is calculated using the Eq. 4 and direction is approximated using the formula given in Eq. 3 the angle theta is calculated using the Eq. 5:

$$
\begin{aligned}
& |G|=\left|G_{x}\right|+\left|G_{y}\right| \\
& M=\sqrt{G_{x}^{2}+G_{y}^{2}} \\
& \theta=\tan ^{-1}\left(\frac{G_{y}}{G_{x}}\right)
\end{aligned}
$$

The iris images are collected from the Institute of Automation, Chinese Academy of Science (CASIA) database. The database contains total of 22051 iris images from more than 700 subjects and 1500 eyes ( $\mathrm{Li}$ and Kang, 2008). These images are used for segmentation and implemented using the MATLAB function. The range of the values of iris is from 90-150 pixels and pupil radius is from 28-75 pixels (Proenca and Alexandre, 2006). The circular Hough transform was performed within the iris region.

Once the iris region is isolated from an eye image, the next stage is to transform the iris region to fixed dimensions in order to allow comparison. The concentric iris image is unwrapped as shown in Fig. 1.

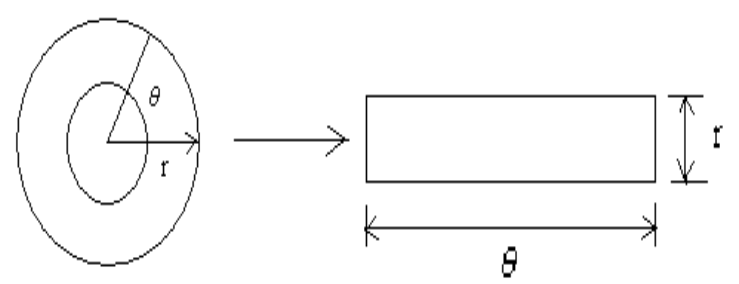

(a)

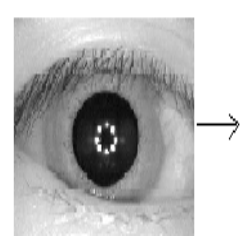

(b)

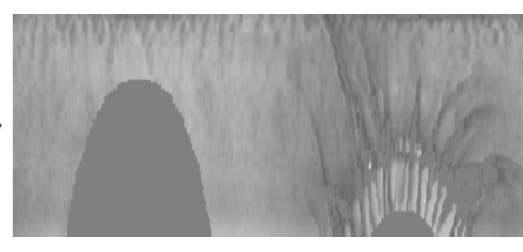

(c)
Fig. 1: (a) Iris unwrapped image (b) Original image (c) Normalized image

The diameter of the pupil and the iris is not constant for all images. It is necessary to transform these to normalize the distance between pupil and iris by using the transform Eq. 7-8.

The mapping of the concentric iris region from (x. y) coordinates to the normalized polar representation is given by the Eq. 6 :

$\mathrm{I}(\mathrm{x}(\mathrm{r}, \theta), \mathrm{y}(\mathrm{r}, \theta)) \rightarrow \mathrm{I}(\mathrm{r}, \theta)$

Where:

$\mathrm{I}(\mathrm{x}, \mathrm{y})=$ Iris image

$(\mathrm{x}, \mathrm{y})=$ Original Cartesian coordinates

$(\mathrm{r}, \theta)=$ Corresponding polar coordinates

The pupil coordinates are $\left(\mathrm{x}_{\mathrm{p}}, \mathrm{y}_{\mathrm{p}}\right)$ and iris coordinates are $\left(\mathrm{X}_{\mathrm{i}}, \mathrm{Y}_{\mathrm{i}}\right)$ along $\theta$ direction:

$\mathrm{x}(\mathrm{r}, \theta)=(1-\mathrm{r}) \mathrm{x}_{\mathrm{p}}(\theta)+\mathrm{rx}_{\mathrm{i}}(\theta)$

$\mathrm{y}(\mathrm{r}, \theta)=(1-\mathrm{r}) \mathrm{y}_{\mathrm{p}}(\theta)+\mathrm{ry}_{\mathrm{i}}(\theta)$

The iris images are first scaled to get constant distance between pupil and iris region. While comparing the two images one is considered as the reference image. Once two images are same dimension, the features are extracted from the iris region by considering the intensity values along with the concentric circles with origin at the center of pupil.Once the normalized iris region is obtained in the $2 \mathrm{D}$ rectangular form, this can be used to extract the features. The iris pattern is demodulated to extract phase information using the $2 \mathrm{D}$ wavelet A 2D Gabor filter for any image $I(x, y)$ is given by the Eq. 9. The particular position in the image is given by 
$\left(\mathrm{x}_{0}, \mathrm{y}_{0}\right)$, width and length is denoted by $(\mathrm{a}, \beta)$, the modulation is denoted by $\left(\mathrm{u}_{0}, \mathrm{v}_{0}\right)$ the complex valued coefficients whose real and imaginary parts specify the coordinates of a pharos in the complex plane (Daugman, 2004). Where the frequency $\mathrm{w}_{0}$ is given by the Eq. 10:

$$
\begin{aligned}
& \mathrm{G}(\mathrm{x}, \mathrm{y})=\mathrm{e}^{-\pi\left[\left(\mathrm{x}-\mathrm{x}_{0}\right)^{2} / \alpha^{2}+\left(\mathrm{y}-\mathrm{y}_{0}\right)^{2} / \beta^{2}\right]} \mathrm{e}^{-2 \pi \mathrm{i}\left[\mathrm{u}_{0}\left(\mathrm{x}-\mathrm{x}_{0}\right)+\mathrm{v}_{0}\left(\mathrm{y}-\mathrm{y}_{0}\right)\right]} \\
& \mathrm{w}_{0}=\sqrt{\mathrm{u}_{0}^{2}+\mathrm{v}_{0}^{2}}
\end{aligned}
$$

The iris pattern is convolved with the modulation and phase Quantization of complex valued Gabor wavelet which is represented by the Eq. 11:

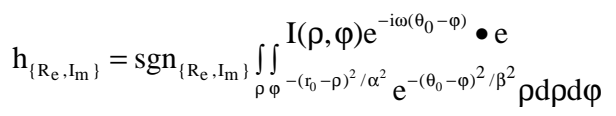

Where:

$$
\begin{aligned}
\mathrm{h}_{\left\{\mathrm{Re}_{\mathrm{e}}, \mathrm{I}_{\mathrm{m}}\right\}}= & \text { Used as complex valued bit whose real } \\
& \text { and imaginary parts are either } 1 \text { or } 0 \\
& \text { depending on the sign of the } 2 \mathrm{D} \text { integral, } \\
& \text { The iris image in polar coordinate system } \\
& \text { is denoted by } \mathrm{I}(\mathrm{P}, \phi) \\
\text { a and } \beta= & \text { Considered as the multi scale } 2 \mathrm{D} \text { wavelet } \\
& \text { size parameters } \\
\omega= & \text { Wavelet frequency } \\
\left(\mathrm{r}_{0}, \theta_{0}\right)= & \text { Represent the polar coordinates of iris } \\
& \text { region }
\end{aligned}
$$

The phase information is used for recognizing the iris whereas amplitude information is used for finding the contrast and camera gain.

After feature extraction, an iris image is converted into feature vectors. The iris template stored is used for matching. Two irises can be compared using the features vectors. The difference of two iris vector is calculated.

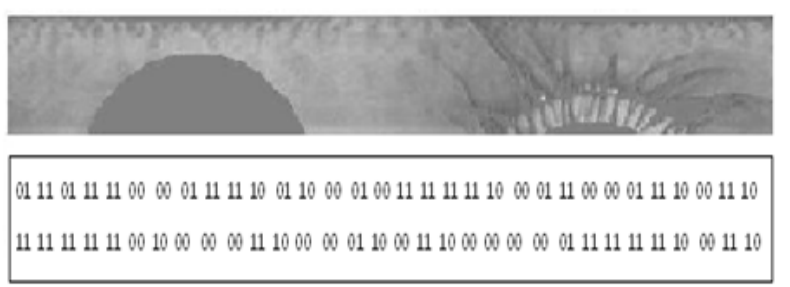

Fig. 2: Sample normalized iris and encoded iris pattern
The proposed matching algorithm, Euclidian Distance can record results for iris images. The comparison is performed intra-class database as well as inter-class database. The results are shown in Table 3-4. The template is used for comparison of different iris patterns. The normalization of the iris region determines the radial and angular resolutions which are to be used for encoding the iris pattern to create the iris template. The values generated from encoding process are given in Fig. 2.

\section{RESULTS AND DISCUSSION}

The performance of iris recognition system is tested at various stages. The tests were performed for image preprocessing, segmentation, normalization, feature extraction and matching.

The images are captured using the system set up with high resolution camera installed in our College Research Laboratory. The collected data base is having total 1000 images of right and left eye with very good image quality and extremely clear iris texture details. These images are collected from different 50 male and female volunteers. Here, we have selected 100 iris images from CASIA database and 100 images from our captured images for testing. The images from CASIA database are standard and selected based on good segmentation results. The iris images from both the datasets were tested for intra-class and interclass comparison given in Table 1 . The results of the segmentation efficiency are given in Table 2.

The segmentation efficiency for our acquired images is less due to the factors affecting for capturing the image like light, distance between the camera and person. The wavelet transform is applied on the normalized iris region to encode, the iris pattern. The Gabor wavelet is convolved with the iris pattern. Table 3 gives the some of the results of Gabor wavelet and Table 4 gives some of the iris feature values.

Table 1: Intra-class and inter-class comparison

\begin{tabular}{llll}
\hline Set name & $\begin{array}{l}\text { Number of } \\
\text { eye images }\end{array}$ & $\begin{array}{l}\text { Intra class } \\
\text { comparison }\end{array}$ & $\begin{array}{l}\text { Intra class } \\
\text { comparison }\end{array}$ \\
\hline CASIA & 100 & 150 & 250 \\
$\begin{array}{l}\text { Images acquired } \\
\text { in our research }\end{array}$ & 100 & 150 & 250 \\
laboratory & & & \\
\hline
\end{tabular}

Table 2: Efficiency for segmentation results

\begin{tabular}{ll}
\hline Set name & Segmentation efficiency \\
\hline CASIA & $92 \%$ \\
Images acquired in our & $72 \%$ \\
research laboratory & \\
\hline
\end{tabular}




\begin{tabular}{|c|c|c|c|c|c|c|}
\hline 0.0000 & 0.0000 & 0.0000 & 0.0000 & 0.0000 & 0.0000 & 0.0000 \\
\hline 0.0000 & 0.0000 & 0.0000 & 0.0000 & 0.0000 & 0.0000 & 0.0000 \\
\hline 0.0001 & 0.0001 & 0.0001 & 0.0002 & 0.0002 & 0.0003 & 0.0004 \\
\hline 0.0006 & 0.0007 & 0.0009 & 0.0012 & 0.0014 & 0.0017 & 0.0021 \\
\hline 0.0025 & 0.0029 & 0.0034 & 0.0040 & 0.0046 & 0.0053 & 0.0061 \\
\hline 0.0070 & 0.0079 & 0.0089 & 0.0099 & 0.0111 & 0.0124 & 0.0137 \\
\hline 0.0151 & 0.0166 & 0.0182 & 0.0199 & 0.0217 & 0.0236 & 0.0256 \\
\hline 0.0227 & 0.0299 & 0.0322 & 0.0346 & 0.0371 & 0.0397 & 0.0424 \\
\hline 0.0452 & 0.0480 & 0.0510 & 0.0541 & 0.0573 & 0.0606 & 0.0640 \\
\hline 0.0675 & 0.0711 & 0.0748 & 0.0785 & 0.0824 & 0.0863 & 0.0904 \\
\hline 0.0945 & 0.0987 & 0.1030 & 0.1074 & 0.1119 & 0.1164 & 0.1210 \\
\hline 0.1257 & 0.1305 & 0.1353 & 0.1403 & 0.1452 & 0.1503 & 0.1554 \\
\hline 0.1606 & 0.1658 & 0.1711 & 0.1765 & 0.1819 & 0.1874 & 0.1929 \\
\hline 0.1985 & 0.2041 & 0.2098 & 0.2155 & 0.2212 & 0.2270 & 0.2329 \\
\hline 0.2387 & 0.2446 & 0.2506 & 0.2566 & 0.2626 & 0.2686 & 0.2746 \\
\hline 0.2807 & 0.2868 & 0.2929 & 0.2991 & 0.3052 & 0.3114 & 0.3176 \\
\hline 0.3238 & 0.3300 & 0.3362 & 0.3425 & 0.3487 & 0.3550 & 0.3612 \\
\hline 0.3675 & 0.3737 & 0.3800 & 0.3862 & 0.3925 & 0.3987 & 0.4049 \\
\hline 0.4112 & 0.4174 & 0.4236 & 0.4298 & 0.4360 & 0.4422 & 0.4483 \\
\hline 0.4545 & 0.4606 & 0.4668 & 0.4729 & 0.4789 & 0.4850 & 0.4911 \\
\hline 0.4971 & 0.5031 & 0.5091 & 0.5150 & 0.5209 & 0.5269 & 0.5327 \\
\hline 0.5386 & 0.5444 & 0.5502 & 0.5560 & 0.5617 & 0.5674 & 0.5731 \\
\hline 0.5788 & 0.5844 & 0.5900 & 0.5955 & 0.6010 & 0.6010 & 0.6010 \\
\hline
\end{tabular}

Table 4: Sample features values

\begin{tabular}{llllll}
\hline 130 & 183 & 108 & 130 & 183 & 57 \\
153 & 133 & 108 & 152 & 132 & 40 \\
185 & 163 & 108 & 182 & 168 & 32 \\
148 & 163 & 110 & 148 & 165 & 57 \\
173 & 175 & 110 & 173 & 178 & 40 \\
183 & 158 & 108 & 180 & 165 & 47 \\
160 & 160 & 108 & 155 & 165 & 55 \\
165 & 188 & 115 & 157 & 190 & 37 \\
163 & 160 & 98 & 158 & 165 & 37 \\
183 & 153 & 103 & 178 & 157 & 43 \\
185 & 163 & 108 & 182 & 168 & 32 \\
183 & 158 & 108 & 180 & 165 & 47 \\
\hline
\end{tabular}

\section{CONCLUSION}

The study "Efficient Feature Extraction Methodology Using wavelet Transform for the Development of Person Identification System Using Iris Biometrics" is focused on feature extraction and encoding of the iris pattern. The efficient and effective method used for feature extraction is Gabor wavelet transform. The iris image normalized to the fixed sized rectangular pattern was encoded to generate iris template. Using the iris template the Euclidian Distance was calculated of each individual iris image. The iris code was used for the comparison of inter-class and intra-class iris pattern. The proposed system is found successful and able to identify a person using his/her iris images. The experimental results show the better performance of the proposed system.

\section{REFERENCES}

Daugman, J., 2004. How iris recognition works. IEEE Trans Circuits Syst. Video Technol., 14: 21-30. DOI: 10.1109/TCSVT.2003.818350
Delac, K. and M. Grgic, 2004. A survey of biometric recognition methods. Proceedings of the 46th International Symposium on Electronics in Marine, Jun. 16-18, IEEE Xplore Press, USA., pp: 184-193. DOI: 10.1109/ELMAR.2004.1356372

Khan, M.K., J. Zhang and S.J. Horng, 2004. An effective iris recognition system for identification of humans. Proceedings of the 8th International Multitopic Conference, Dec. 24-26, IEEE Xplore Press, USA., pp: 114-117. DOI: 10.1109/INMIC.2004.1492856

Lewis, J.W., 2002. Biometrics for secure Identity Verification: Trends and Developments. M.Sc. Thesis, University of Maryland, Bowie State University. http://acsupport.europe.umuc.edu/ meinkej/inss690/lewis.p df

Li, Y. and S.B. Kang, 2008. Chinese Academy of Science. ScientificCommons.

Ma, L., Y. Wang and T. Tan, 2002. Iris recognition based on multichannel Gabor filtering. Proceedings of the International 5th Asian Conference on Computer Vision, Jan. 23-25, Melbourne, Australia, pp: 279-283. http://citeseerx.ist.psu.edu/viewdoc/download?doi= 10.1.1.132.3967\&rep=rep1\&type $=$ pdf

Masek, L., 2003. The University of Western Australia. Recognition of human iris patterns for biometric identification. The University of Western Australia. http://citeseerx.ist.psu.edu/viewdoc/download?doi= 10.1.1.90.5112\&rep=rep1\&type $=$ pdf 
Proenca, H. and L.A. Alexandre, 2006. A method for the identification of inaccuracies in pupil segmentation. Proceedings of the 1st International Conference on Availability Reliability and Security, Apr. 20-22, IEEE Xplore Press, pp: 5-5. DOI: 10.1109/ARES.2006.9

Sonkamble, S., R. Thool and B. Sonkamble, 2010. Survey of biometric recognition systems and their applications. J. Theoretical Applied Inform. Technol., 45-51. http://www.jatit.org/volumes/researchpapers/Vol11No1/6Vol11No1.pdf
Sonkamble, S., R.B. Thool, A. Sonkamble, 2008. An effective machine-vision system for information security and privacy using iris biometrics. Information Technology, MMCOE. http://www.iiis.org/CDs2008/CD2008SCI/SCI200 8/PapersPdf/S046RO.pdf

Wang, Y. and J.Q. Han, 2005. Iris recognition using independent component analysis. Proceedings of the International Conference on Machines Learning and Cybernatics, Aug. 18-21, IEEE Xplore Press, Guangzhou, China, pp: 4487-4492. DOI: 10.1109/ICMLC.2005.1527729 